\title{
Multiplicity of Cournot Equilibria and Involuntary Unemployment
}

\author{
Leo Kaas*
}

\begin{abstract}
In an overlapping generations model with Cournot competition on the goods market it is shown that a continuum of stationary states and perfect foresight trajectories exists with unemployment at arbitrary low wages. Decisive for this is the influence that different forecast functions have on the objective demand curve, even though they are consistent with perfect foresight. With an example it is shown that simple adaptive and constant memory forecast rules generate such unemployment equilibria. The corresponding temporary equilibrium dynamics may display stable unemployment and unstable full employment equilibria. Journal of Economic Literature Classification Numbers: D43, D51, E24.
\end{abstract}

\section{INTRODUCTION}

The possibility of involuntary unemployment in simple three commodity macroeconomic models with Cournot competition on the goods market has been investigated intensively. The main question is whether unemployment is necessarily due to wage rigidities forced by union power, or may prevail even at a perfectly competitive labor market in which case the wage rate would be zero. All this work goes back to the influential article of Hart [9] which applies the objective demand approach in an atemporal setting, but excludes involuntary unemployment by an ad-hoc assumption on the marginal revenue curve. Many articles discuss different assumptions leading to involuntary unemployment or not (see the surveys of Silvestre [14, 15]).

Some authors have applied the ideas of Hart in an intertemporal framework where money is used as a store of value and therefore price expectations are becoming important. Dehez [5] points out the possibility of involuntary unemployment in the monopolistic case with full incorporation of all income feedback (or Ford) effects under very general conditions on price expectations. In an overlapping generations model Schultz [13]

* The author thanks V. Böhm, T. Hens, A. Stiefenhofer, an associate editor, and especially P. Madden for many helpful comments. 
excludes involuntary unemployment under rational expectations, or more precisely under inelastic price expectations of households, where firms disregard the income feedback effects generated by their own strategies. D’Aspremont, Dos Santos Ferreira, and Gérard-Varet [4] show on the other hand that involuntary unemployment can arise if the income feedback effects are taken into account.

In this paper I question the assumption of Schultz on inelastic price expectations. While Schultz himself notes that his result depends crucially on the inelasticity of price expectations (p. 69/70), it is not clear why in a rational expectations equilibrium (which should in a deterministic framework be equivalent to a perfect foresight equilibrium) price expectations are inelastic. If one regards a rational expectations equilibrium as the asymptotic outcome of a learning process, as it is done in most of the learning literature (see, e.g., Marcet and Sargent [11]), price inelastic rational expectations can only be justified by price inelastic learning processes. That is, price expectations for the next period must not depend on the actual price level, which is a quite strong assumption. Furthermore, as shown by Lettau and Van Zandt [10], steady states in competitive OLG models may be stable under adaptive expectations only if agents include the current price level in their forecast function.

A dependence of imperfectly competitive equilibria on price expectations has been recognized by Rankin [12]. He shows by consideration of first order conditions that in a model with perfect competition on the goods market and Cournot competition on the labor market multiple steady states (with no forecast errors) may occur. Rankin argues, on the other hand, that perfect foresight steady states are unique, since perfect forecasts are inelastic in the actual price, if a sector structure similar to the one in Hart [9] is assumed and consumers at one sector are dispersed among the other sectors in the second period of their life. However, this of course needs not be the case if consumers do not alter their consumption habits over time and expect therefore an influence of the observed sector price on the price level tomorrow.

The main result proven in this paper is that under very general conditions on the utility function, but by allowing expectations to depend on the actual price level, there will exist a continuum of stationary equilibria with employment below the full employment level at arbitrary low real wages. Moreover, within a certain interval nearly arbitrary perfect foresight trajectories are possible. As should be the case, it is furthermore shown that the interval containing perfect foresight equilibria converges to the full employment allocation if the number of firms tends to infinity.

The paper is organized as follows: The next section introduces the model and the employed equilibrium concept, while Section 3 contains the main theorems. Since there is no general class of forecast functions which 
together with standard assumptions on the utility function yield existence of equilibria, we have to construct forecast functions which may seem too arbitrary. However, the crucial parameter for the occurrence of unemployment is the elasticity with respect to the actual price, which has different values for various standard forecast functions. As an illustration, Section 4 contains an example with a CES utility function, where a simple class of constant elasticity price expectations (containing log-linear adaptive and finite memory expectations) leads to a continuum of unemployment steady states. Additionally, the corresponding temporary equilibrium dynamics shows that unemployment equilibria may be stable, while full employment equilibria are not.

\section{THE MODEL}

Consider an economy which evolves in discrete time periods $t=0,1,2, \ldots$. In each period there are three commodities: labor, a consumption good and money. There are $n \geqslant 2$ identical firms producing the consumption good by means of labor input. The price of the consumption good is $p_{t}$, the wage rate is $w_{t}$ and the price of money is normalized to one. Wage rates are taken as given by all agents in the economy, but their determination is left undescribed. In the case of a competitive labor market, they are either at a labor market clearing level or they are zero, if there is involuntary unemployment. On the goods market there is Cournot competition.

The household sector is described by an overlapping generations structure, namely one representative young and one old agent in each period. The young household supplies inelastically a fixed amount of labor $\bar{L}>0$ and receives wage as well as profit income from all firms. Households have preferences over consumption plans $\left(x_{t}, x_{t+1}\right)$ in both periods of their life described by an intertemporal utility function $u: \mathbb{R}_{+}^{2} \rightarrow \mathbb{R}$.

Assumption 1. $u$ is $C^{2}$, homothetic, strictly quasi-concave and strictly monotonic on $\mathbb{R}_{++}^{2} \cdot u_{i}{ }^{\prime}\left(x_{1}, x_{2}\right) \rightarrow \infty$ if $x_{i} \rightarrow 0, x_{j}>0, j \neq i$, for $i=1,2$.

This implies that the utility maximization problem of a young household at income $I>0$, price $p>0$ and expected price $p^{\mathrm{e}}>0$ leads to a first-period consumption demand

$$
x^{*}=c\left(\frac{p^{\mathrm{e}}}{p}\right) \frac{I}{p}
$$

with a continuously differentiable propensity to consume $c: \mathbb{R}_{++} \rightarrow(0,1)$. 
For a full description of the objective demand curve, it is very important how price expectations are formed. We assume that price expectations are described by a sequence of continuous forecast functions $\Psi=\left(\Psi_{t}\right)_{t \geqslant 0}$, where each $\Psi_{t}: \mathbb{R}_{++}^{t+1} \rightarrow \mathbb{R}_{++}$maps current and past prices $\left(p_{t}, \ldots, p_{0}\right)$ into the for $t+1$ expected price $p_{t+1}^{\mathrm{e}}$. The forecast function summarizes how people extract information from past data. There are no further conditions on $\Psi_{t}$ imposed, since we will concentrate on trajectories with perfect foresight where the corresponding forecast functions fulfill already the strongest consistency criterion one can think of. As long as there is no reason for confusion, we will write $\psi_{t}():. \mathbb{R}_{++} \rightarrow \mathbb{R}_{++}$instead of $\Psi_{t}\left(., p_{t-1}, \ldots, p_{0}\right)$.

The old household in period 0 holds an amount $M>0$ of money. We assume no government activity which together with goods (and money) market clearing implies that $M$ is the stock of money held by all subsequent old generations. This implies that in every period the aggregate demand for the consumption good at income $I$ and forecast function $\psi$ is given by the continuous function

$$
D_{\psi}(p, I):=c\left(\frac{\psi(p)}{p}\right) \frac{I}{p}+\frac{M}{p} .
$$

It is easy to see that

$$
\lim _{p \rightarrow 0} D_{\psi}(p, I)=\infty \quad \text { and } \quad \lim _{p \rightarrow \infty} D_{\psi}(p, I)=0,
$$

so that there exists a (not necessarily unique and continuous) inverse mapping $P_{\psi}(., I)$, i.e.

$$
P_{\psi}(Y, I) \in\left\{p>0 \mid Y=D_{\psi}(p, I)\right\} \quad \forall Y>0, \quad I>0 .
$$

The $n$ firms produce the consumption good from labor with identical labor requirement function $l: \mathbb{R}_{+} \rightarrow \mathbb{R}_{+}$which fulfills

Assumption 2. $\quad l$ is $C^{2}$ and satisfies $l^{\prime}>0, l^{\prime \prime} \geqslant 0$ and $l(0)=0$.

Firms act as Cournot oligopolists against the objective inverse demand $P_{\psi}$ and are assumed to take the wage rate and the income as given, i.e. they ignore that their strategic choice affects the income of the young consumer. Alternatively, one can think of an economy consisting of a large number of identical sectors, where each household who receives income in a sector consumes in some other sector and with consumers being uniformly distributed (Hart [9], Schultz [13]). The overall equilibrium in one sector of such an economy corresponds then to the equilibrium defined below. The possible incorporation of income feedback effects facilitates involuntary 
unemployment even at inelastic price expectations (d'Aspremont, Dos Santos Ferreira, and Gérard-Varet [4]), but should allow similar multiplicity results.

Before defining a symmetric Cournot equilibrium, we denote by

$$
\hat{y}(y):=l^{-1}(\bar{L}-(n-1) l(y))
$$

the maximum output of a single firm who takes the demand constraint on the labor market $\bar{L}$ and the output of all the other firms $y$ as given.

Definition 1. Let $w \geqslant 0$ and $\psi \in C\left(\mathbb{R}_{++}\right)$. A temporary symmetric Cournot equilibrium relative to $(w, \psi)$ is a tuple $(y, p, I)$ such that

$$
\begin{aligned}
\text { (i) } y & \in \operatorname{argmax}\left\{y^{\prime} P_{\psi}\left((n-1) y+y^{\prime}, I\right)-w l\left(y^{\prime}\right) \mid 0 \leqslant y^{\prime} \leqslant \hat{y}(y)\right\}, \\
\text { (ii) } p & =P_{\psi}(n y, I), \\
\text { (iii) } I & =n p y .
\end{aligned}
$$

The set of all temporary symmetric Cournot equilibria relative to $(w, \psi)$ is denoted $\operatorname{TSCE}(w, \psi)$.

Notice that the concentration on symmetric equilibria is not too restrictive, since all unemployment equilibria must be symmetric if $P_{\psi}$ is differentiable with a negative slope at any aggregate equilibrium output $Y=\sum_{i=1}^{n} y_{i}$ : the first order conditions $y_{i} P_{\psi}{ }^{\prime}(Y, I)+P_{\psi}(Y, I)=w l^{\prime}\left(y_{i}\right)$, $i=1, \ldots, n$, can only be fulfilled if all $y_{i}$ are identical, since the left hand side is strictly decreasing in $y_{i}$, while the right hand side is non-decreasing.

If $\psi$ is differentiable, an easy computation yields

$$
\frac{D_{\psi}^{\prime}(p, I) p}{D_{\psi}(p, I)}=-1+\frac{c\left(\frac{\psi(p)}{p}\right) I\left(1-c\left(\frac{\psi(p)}{p}\right)\right)\left(\sigma\left(\frac{\psi(p)}{p}\right)-1\right)\left(\eta_{\psi}(p)-1\right)}{c\left(\frac{\psi(p)}{p}\right) I+M}
$$

where

$$
\sigma(\theta):=\frac{d}{d \theta}\left(\frac{\theta c(\theta)}{1-c(\theta)}\right) \frac{1-c(\theta)}{c(\theta)}=1+\frac{c^{\prime}(\theta) \theta}{c(\theta)(1-c(\theta))} \geqslant 0
$$

is the intertemporal elasticity of substitution and $\eta_{\psi}(p):=\psi^{\prime}(p) p / \psi(p)$ the elasticity of the forecast function. Since in a temporary equilibrium the income is determined by $I=M /(1-c(\psi(p) / p))$ one gets

$$
\frac{D_{\psi}^{\prime}(p, I) p}{D_{\psi}(p, I)}=-1+c\left(\frac{\psi(p)}{p}\right)\left(1-c\left(\frac{\psi(p)}{p}\right)\right)\left(\sigma\left(\frac{\psi(p)}{p}\right)-1\right)\left(\eta_{\psi}(p)-1\right) .
$$


Therefore, $D_{\psi}$ and hence $P_{\psi}$ is differentiable with negative slope in an equilibrium, if, for instance, $(\sigma(\theta)-1)\left(\eta_{\psi}(p)-1\right)<4$ uniformly in $\theta$ and $p$.

For $(y, p, I) \in \operatorname{TSCE}(w, \psi)$ and a continuously differentiable inverse demand function, the first order condition of (i) in Definition 1 is

$$
p\left(1+\frac{D_{\psi}(p, I)}{n p D_{\psi}^{\prime}(p, I)}\right) \geqslant w l^{\prime}(y) \quad \text { with equality if } \quad n l(y)<\bar{L} .
$$

Using (2), this is equivalent to

$$
\begin{gathered}
1-c\left(\frac{\psi(p)}{p}\right)\left(1-c\left(\frac{\psi(p)}{p}\right)\right)\left(\sigma\left(\frac{\psi(p)}{p}\right)-1\right)\left(\eta_{\psi}(p)-1\right) \\
\geqslant \frac{p}{n\left(p-w l^{\prime}(y)\right)} \quad \text { with equality if } n l(y)<\bar{L} .
\end{gathered}
$$

From this condition an extension of the result of Schultz (1992, Theorem 1) on the impossibility of involuntary unemployment can be derived: If $(\sigma()-1).(\eta()-1)<$.2 uniformly (which holds under the Schultz assumption $\eta(.) \equiv 0)$, there must be full employment for sufficiently low wages, since the left hand side of $(3)$ is greater than $1 / 2$, while the right hand side converges to $1 / n \leqslant 1 / 2$ for $w \rightarrow 0$. Therefore, the inequality must be strict for low wages which requires full employment. On the other hand, it seems clear that for arbitrary preferences and forecast functions (3) may be fulfilled with equality, and hence unemployment at arbitrary low wages can not be excluded. That this phenomenon can even be consistent with perfect foresight will be shown in the next section. First, we need the definition of perfect foresight equilibria:

Definition 2. Let $\left(w_{t}\right)_{t \geqslant 0} \geqslant 0$ be a sequence of non-negative wages. A sequence $\left(\left(y_{t}, p_{t}, I_{t}\right)\right)_{t \geqslant 0}$ is a perfect foresight symmetric Cournot equilibrium relative to $\left(w_{t}\right)_{t \geqslant 0}$ if there exists $\Psi=\left(\Psi_{t}\right)_{t \geqslant 0}, \Psi_{t} \in C\left(\mathbb{R}_{++}^{t+1}, \mathbb{R}_{++}\right)$ $\forall t \geqslant 0$, such that for all $t \geqslant 0$

(i) $\left(y_{t}, p_{t}, I_{t}\right) \in \operatorname{TSCE}\left(w_{t}, \psi_{t}\right)$,

(ii) $\psi_{t}\left(p_{t}\right)=\Psi_{t}\left(p_{t}, p_{t-1}, \ldots, p_{0}\right)=p_{t+1}$.

Notice that the above definition of a perfect foresight equilibrium needs the concept of forecast functions. In this regard the model differs from competitive temporary equilibrium models described by relations of the form $T\left(x_{t}, x_{t+1}^{\mathrm{e}}\right)=0$ (see for example Grandmont [7]), where a perfect foresight equilibrium is simply defined as a sequence $\left(x_{t}\right)_{t \geqslant 0}$ solving $T\left(x_{t}, x_{t+1}\right)=0$ for all $t$. There are many forecast functions which generate a perfect foresight equilibrium as a sequence of temporary equilibria and which differ 
in their expectations outside the equilibrium path. Since these off-equilibrium expectations do not matter in a competitive model, a perfect foresight equilibrium can be defined independently of a forecast function.

In contrast to this, in an imperfectly competitive model the off-equilibrium expectations have to be specified explicitly since they determine the objective demand curve against which oligopolists maximize. However, expectations outside the equilibrium cannot be determined by a rationality requirement, since an agent who uses some forecast rule $\Psi$ which fulfills the perfect foresight condition (ii) will be completely satisfied with this rule and has no reason to change it.

Finally, the definition of a stationary equilibrium is straightforward:

Definition 3. A stationary symmetric Cournot equilibrium relative to $w \geqslant 0$ is a tuple $(y, p, I)$ such that there exists $\psi \in C\left(\mathbb{R}_{++}\right)$with

(i) $(y, p, I) \in \operatorname{TSCE}(w, \psi)$,

(ii) $\psi(p)=p$.

\section{MULTIPLICITY OF PERFECT FORESIGHT EQUILIBRIA}

It will be shown first that fixing the expected inflation rate is not sufficient to obtain unique temporary symmetric Cournot equilibria. Instead, a continuum of equilibria with employment below the full employment level can occur at arbitrarily low wages. The only required assumption is an intertemporal elasticity of substitution different from 1, i.e. expectations have to matter locally.

THEOREM 1. Let $\theta>0$ and $\sigma(\theta) \neq 1$. Then there exist $\bar{w}>0$ and $\tilde{y}<\bar{y}:=l^{-1}(\bar{L} / n)$, such that for all $y \in[\tilde{y}, \bar{y}], p=M /(n y(1-c(\theta)))$, and all $0 \leqslant w \leqslant \bar{w}$, there exists $\psi \in C\left(\mathbb{R}_{++}\right)$with $(y, p, n p y) \in \operatorname{TSCE}(w, \psi)$ and $\psi(p)=\theta p$. Moreover, if $u$ is $C^{3}$, then $\bar{w}$ and $\tilde{y}$ depend continuously on $\theta$.

Proof. $\sigma(\theta) \neq 1$ implies $c^{\prime}(\theta) \neq 0$, and there exist $\underline{c}<c(\theta)<\bar{c}, \underline{\theta}<\theta<\bar{\theta}$ such that $c$ maps $[\underline{\theta}, \bar{\theta}]$ diffeomorphically onto $[\underline{c}, \bar{c}]$. If $u$ is $C^{3}, c$ is $C^{2}$ and $\underline{c}$ and $\bar{c}$ may be chosen to depend continuously on $\theta$ (see Abraham, Marsden, and Ratiu [1, p. 105/106]). Define

$$
\begin{aligned}
& \bar{w}:=M(1-1 / n)\left(n \bar{y} l^{\prime}(\bar{y})(1-c(\theta))\right)^{-1}, \\
& \tilde{y}:=\min \left\{\begin{array}{ll}
y \geqslant 0 \mid \begin{array}{l}
\frac{n-1}{n}+\frac{1}{n}\left(\frac{\hat{y}(y)}{y}\right)^{(n-1) / n} \\
\frac{n-1}{n} \frac{y}{\hat{y}(y)}+\frac{1}{n}
\end{array} & \geqslant 1-c(\theta)+\bar{c}
\end{array}\right\} .
\end{aligned}
$$


Notice that $\tilde{y}<\bar{y}$ since both inequalities hold strictly for $y=\bar{y}$ and that $\tilde{y}$ is continuous in $\theta$ if $\underline{c}$ and $\bar{c}$ are continuous in $\theta$ since $y \mapsto \hat{y}(y) / y$ is strictly monotonically decreasing.

Let $0 \leqslant w \leqslant \bar{w}$ and $\tilde{y} \leqslant y \leqslant \bar{y}$. Set

$$
p:=\frac{M}{n y(1-c(\theta))} \quad \text { and } \quad \eta:=1-\frac{w l^{\prime}(y)}{p} \geqslant \frac{1}{n}
$$

Define $f:[0, \hat{y}(y)] \rightarrow \mathbb{R}_{++}$by

$$
f\left(y^{\prime}\right):= \begin{cases}\frac{n p y}{y^{\prime}+(n-1) y}, & 0 \leqslant y^{\prime} \leqslant y, \\ \frac{p y}{y^{\prime \eta} y^{1-\eta}}, & y \leqslant y^{\prime} \leqslant \hat{y}(y) .\end{cases}
$$

Clearly, $f$ is continuous and strictly monotonically decreasing and $f(y)=p$. Define $\underline{d}:=1+\underline{c}(1-c(\theta))^{-1}$ and $\bar{d}:=1+\bar{c}(1-c(\theta))^{-1}$. We claim that

$$
\underline{d} \frac{M}{y^{\prime}+(n-1) y} \leqslant f\left(y^{\prime}\right) \leqslant \bar{d} \frac{M}{y^{\prime}+(n-1) y} \quad \forall y^{\prime} \in[0, \hat{y}(y)] .
$$

Both inequalities clearly hold for $y^{\prime} \leqslant y$ since $\underline{d} M<n p y=M(1-c(\theta))^{-1}$ $<\bar{d} M$. For $y \leqslant y^{\prime} \leqslant \hat{y}(y)$ the inequalities follow from the estimations

$$
\begin{aligned}
\frac{(n-1) y+y^{\prime}}{n y^{1-\eta} y^{\prime \eta}} & \geqslant \frac{n-1}{n} \frac{y}{\hat{y}(y)}+\frac{1}{n} \\
& \geqslant 1-c(\theta)+\underline{c}=\underline{d}(1-c(\theta))=\underline{d} \frac{M}{n p y} \text { and } \\
\frac{(n-1) y+y^{\prime}}{n y^{1-\eta} y^{\prime \eta}} & \leqslant \frac{n-1}{n}+\frac{1}{n}\left(\frac{\hat{y}(y)}{y}\right)^{1-1 / n} \\
& \leqslant 1-c(\theta)+\bar{c}=\bar{d}(1-c(\theta))=\bar{d} \frac{M}{n p y} .
\end{aligned}
$$

Next we show that $y$ maximizes $\pi\left(y^{\prime}\right):=f\left(y^{\prime}\right) y^{\prime}-w l\left(y^{\prime}\right)$ on $[0, \hat{y}(y)]$. Denote by $\pi_{-}^{\prime}\left(\pi_{+}^{\prime}\right.$ resp.) the left (right resp.) derivative of $\pi$. The claim follows from 


$$
\begin{aligned}
\pi_{-}^{\prime}\left(y^{\prime}\right) & =f\left(y^{\prime}\right)\left(1-\frac{y^{\prime}}{(n-1) y+y^{\prime}}\right)-w l^{\prime}\left(y^{\prime}\right) \\
& \geqslant p(1-1 / n)-\bar{w} l^{\prime}(\bar{y}) \\
& =\frac{(n-1) M}{n^{2}(1-c(\theta))}\left(\frac{1}{y}-\frac{1}{\bar{y}}\right) \geqslant 0, \quad y^{\prime} \leqslant y, \\
\pi_{+}^{\prime}\left(y^{\prime}\right) & =f\left(y^{\prime}\right)(1-\eta)-w l^{\prime}\left(y^{\prime}\right) \\
& \leqslant f(y)(1-\eta)-w l^{\prime}(y)=0, \quad y^{\prime} \geqslant y .
\end{aligned}
$$

Choose any strictly monotonically decreasing $P \in C\left(\mathbb{R}_{++}\right)$such that

$$
\begin{array}{ll}
\underline{d} \frac{M}{Y} \leqslant P(Y) \leqslant \bar{d} \frac{M}{Y} & \forall Y>0 \quad \text { and } \\
P(Y)=f(Y-(n-1) y) & \forall Y \in[(n-1) y,(n-1) y+\hat{y}(y)] .
\end{array}
$$

$P$ has a continuous inverse $D$ satisfying

$$
\underline{d} \frac{M}{p} \leqslant D(p) \leqslant \bar{d} \frac{M}{p} \quad \forall p>0 .
$$

Defining

$$
\psi\left(p^{\prime}\right):=p^{\prime} c^{-1}\left((1-c(\theta))\left(p^{\prime} D\left(p^{\prime}\right) / M-1\right)\right), \quad p^{\prime}>0,
$$

we have $D_{\psi}(., n p y)=D($.$) and \psi(p)=\theta p$. Furthermore, $y$ is a symmetric Cournot equilibrium against the inverse demand $P_{\psi}(., n p y)=P($.$) and$ $P(n y)=p$. Hence, $(y, p, n p y) \in \operatorname{TSCE}(w, \psi)$.

The central part of the proof of Theorem 1 is the construction of a forecast function yielding the existence of the desired temporary equilibrium. Such a forecast function may be quite arbitrary and seem possibly implausible. The decisive reason for this is the fact, that existence of Cournot equilibria with objective demand in general equilibrium models does usually not follow from standard assumptions on the characteristics of the economy (cf. Bonanno [3]). In particular, a restriction to certain classes of forecast functions would require strong assumptions on the inverse demand function which would possibly result in implausible assumptions on the utility function. In this way, Theorem 1 might be a version of a more general multiplicity result that uses more restrictive forecast functions by imposing stronger conditions on $u$. In the next section it will be shown that the same multiplicity phenomenon occurs for the CES utility function and a class of constant elasticity forecast functions. 
The next two corollaries apply Theorem 1 to get multiple perfect foresight and stationary equilibria.

COROLlaRy 1. Let $K \subset \mathbb{R}_{++} \backslash \sigma^{-1}(1)$ be compact and assume $u$ to be $C^{3}$. Then there exist $\bar{w}>0$ and $\tilde{y}<\bar{y}$, such that for all $\left(w_{t}\right)_{t \geqslant 0}$ with $0 \leqslant w_{t} \leqslant \bar{w} \forall t \geqslant 0$ all sequences $\left(\left(y_{t}, p_{t}, n p_{t} y_{t}\right)\right)_{t \geqslant 0}$ satisfying

$$
y_{t}=\frac{M}{n p_{t}\left(1-c\left(p_{t+1} / p_{t}\right)\right)} \in[\tilde{y}, \bar{y}] \quad \text { and } \quad \frac{p_{t+1}}{p_{t}} \in K \quad \forall t \geqslant 0
$$

are perfect foresight symmetric Cournot equilibria relative to $\left(w_{t}\right)_{t \geqslant 0}$.

Proof. Theorem 1 yields continuous functions $\bar{w}(\theta)$ and $\tilde{y}(\theta)$ for $\theta \in K$. Define

$$
\bar{w}:=\inf \{\bar{w}(\theta) \mid \theta \in K\}>0 \quad \text { and } \quad \tilde{y}:=\sup \{\tilde{y}(\theta) \mid \theta \in K\}<\bar{y} .
$$

Given $\left(w_{t}\right)_{t \geqslant 0}$ and $\left(\left(y_{t}, p_{t}, n p_{t} y_{t}\right)\right)_{t \geqslant 0}$ as in Corollary 1, Theorem 1 gives the existence of $\psi_{t} \in C\left(\mathbb{R}_{++}\right)$such that $\left(y_{t}, p_{t}, n p_{t} y_{t}\right) \in \operatorname{TSCE}\left(w, \psi_{t}\right)$ and $\psi_{t}\left(p_{t}\right)=p_{t+1}$ for all $t \geqslant 0$. Choose then any $\Psi_{t} \in C\left(\mathbb{R}_{++}^{t+1}, \mathbb{R}_{++}\right)$so that $\Psi_{t}\left(., p_{t-1}, \ldots, p_{0}\right)=\psi_{t}($.$) .$

Corollary 2. Let $\sigma(1) \neq 1$. Then there exist $\bar{w}>0$ and $\tilde{y}<\bar{y}$ such that each $(y, p, n p y)$ with $\tilde{y} \leqslant y \leqslant \bar{y}$ and $p=M /(n y(1-c(1)))$ is a stationary symmetric Cournot equilibrium relative to every $w \in[0, \bar{w}]$.

It can be easily shown that in the perfectly competitive case where firms are price takers and the price is determined by goods market equilibrium, unemployment does not prevail at arbitrarily low wage rates, irrespective of the forecast function. Therefore, one would expect that, if the number of firms tends to infinity, the interval of possible equilibrium allocations converges to the full employment allocation at sufficiently low wage rates. The result is contained in the following theorem.

THEOREM 2. Let $\hat{L}<\bar{L}$ and $\theta>0$. There exist $N>0$ and $\bar{w}(n)>0, n>N$, so that for all $n>N, 0 \leqslant w \leqslant \bar{w}(n), \psi \in C\left(\mathbb{R}_{++}\right)$, and $(y, p, I) \in \operatorname{TSCE}(w, \psi)$ with $\psi(p)=\theta p$ it follows $n l(y)>\hat{L}$. Furthermore, $N$ and $\bar{w}(n)$ are continuous in $\theta$.

Proof. Assume $(y, p, I) \in \operatorname{TSCE}(w, \psi)$ for some $\psi$ with $\psi(p)=\theta p$. Then the income is determined by $I=M /(1-c(\theta))$. Define $d:=(1+c(\theta))^{-1}+1$ and observe that

$$
\frac{M}{Y}<P_{\psi}(Y, I)<d \frac{M}{Y} \quad \forall Y>0
$$


Choose

$$
\begin{aligned}
N & :=\bar{L} l\left(l^{-1}(\bar{L}-\hat{L}) d^{-1}\right)^{-1} \quad \text { and } \\
\bar{w}(n) & :=\frac{M\left(l^{-1}(\bar{L}-\hat{L})-d l^{-1}(\bar{L} / n)\right)}{n \bar{L} l^{-1}(\bar{L} / n)}>0, \quad n>N .
\end{aligned}
$$

Assume now $n>N, w \leqslant \bar{w}(n)$, and suppose $n l(y)==: L \leqslant \hat{L}$. From $(y, p, I) \in \operatorname{TSCE}(w, \psi)$ it follows that no firm gains from deviating to the full employment level, i.e. that

$$
\begin{aligned}
P_{\psi}((n-1) y+\hat{y}(y), I) \hat{y}(y)-P_{\psi}(n y, I) y \leqslant w(\bar{L}-(n-1) l(y))-w l(y) \\
=w(\bar{L}-L)
\end{aligned}
$$

Using both inequalities in (4), (5) implies

$$
\frac{n \hat{y}(y)}{(n-1) y+\hat{y}(y)}<\frac{n w}{M}(\bar{L}-L)+d \text {. }
$$

Using the monotonicity and concavity of $l^{-1}$, the left hand side can be estimated to below against $l^{-1}(\bar{L}-L) / l^{-1}(\bar{L} / n)$, so that it follows

$$
l^{-1}(\bar{L}-L)<\left(\frac{n w}{M}(\bar{L}-L)+d\right) l^{-1}(\bar{L} n) .
$$

But this implies

$$
w>\frac{M\left(l^{-1}(\bar{L}-L)-d l^{-1}(\bar{L} / n)\right)}{(\bar{L}-L) n l^{-1}(\bar{L} / n)} \geqslant \bar{w}(n),
$$

a contradiction.

Corollary 3. Let $\hat{L}<\bar{L}, K \subset \mathbb{R}_{++}$compact. Then there exist $N>0$ and $\bar{w}(n), n>N$, so that given $n>N$ and $\left(w_{t}\right)_{t \geqslant 0}$ with $0 \leqslant w_{t} \leqslant \bar{w}(n) \forall t \geqslant 0$, all perfect foresight symmetric Cournot equilibria relative to $\left(w_{t}\right)_{t \geqslant 0}$ with inflation factors contained in $K$ have higher employment than $\hat{L}$ in every period.

Corollary 4. Let $\hat{L}<\bar{L}$. Then there exist $N>0$ and $\bar{w}(n), n>N$, so that given $n>N$ and $0 \leqslant w \leqslant \bar{w}(n)$, all stationary symmetric Cournot equilibria relative to $w$ have higher employment than $\hat{L}$. 


\section{A CES EXAMPLE}

We consider in this section the special case where $u$ is a utility function with constant elasticity of substitution $0 \leqslant \sigma \neq 1$ and discount factor equal to one, which implies $c(\theta)=\left(1+\theta^{1-\sigma}\right)^{-1}$. Furthermore, marginal costs are constant and normalized to 1 , i.e. $l(y)=y$. The class of forecast functions is of the type

$$
\Psi_{t}\left(p_{t}, \ldots, p_{0}\right)=p_{t}^{1+\beta} \Phi_{t}\left(p_{t-1}, \ldots, p_{0}\right)
$$

with some $\beta \in \mathbb{R}$ and continuous $\Phi_{t}$. This class contains log-linear adaptive price or inflation factor expectations

$$
\begin{array}{ll}
p_{t+1}^{\mathrm{e}}=p_{t}^{1+\beta}\left(p_{t}^{\mathrm{e}}\right)^{-\beta}, & -1<\beta \leqslant 0, \\
\frac{p_{t+1}^{\mathrm{e}}}{p_{t}}=\left(\frac{p_{t}}{p_{t-1}}\right)^{\beta}\left(\frac{p_{t}^{\mathrm{e}}}{p_{t-1}}\right)^{1-\beta}, & 0<\beta \leqslant 1,
\end{array}
$$

and constant memory autonomous forecast rules of the type

$$
\Psi_{t}\left(p_{t}, \ldots, p_{0}\right)=p_{t}^{1+\beta} \Phi\left(p_{t-1}, \ldots, p_{t-\tau}\right), \quad t \geqslant \tau .
$$

The following result shows that under this specification there exists a continuum of stationary Cournot equilibria with unemployment at arbitrarily low wage rates. Due to many simplifying estimations in the proof the assumptions are much stronger than needed.

RESUlt 1. Assume $n \leqslant 14$. Then there exists $\tilde{y}<\bar{y}$ such that each $(y, 2 M /(n y), 2 M)$ with $y \in[\tilde{y}, \bar{y}]$ is a stationary symmetric Cournot equilibrium relative to every $0 \leqslant w \leqslant(n-1) 2 M /(n \bar{L})$ for some expectations elasticity $\beta$.

To prove Result 1 we make use of the following Lemma which holds under the general assumptions of Section 2.

Lemma. Let $p>0, y:=M /(n p(1-c(\psi(p) / p)))$, and $I:=n p y$. Assume

(i) $D_{\psi}$ is continuously differentiable and $D_{\psi}{ }^{\prime}(., I)<0$,

(ii) $\operatorname{MR}\left(p^{\prime}\right):=p^{\prime}\left(1+D_{\psi}\left(p^{\prime}, I\right) / n p^{\prime} D_{\psi}^{\prime}\left(p^{\prime}, I\right)\right)$ is strictly monotonically increasing for $p_{h} \geqslant p^{\prime} \geqslant p_{l}$ with $D_{\psi}\left(p_{h}, I\right)=(n-1)$ y and $D_{\psi}\left(p_{l}, I\right)=$ $(n-1) y+\hat{y}(y)$,

(iii) $(y, p)$ satisfies $(3)$.

Then $(y, p, I) \in \operatorname{TSCE}(w, \psi)$. 
Proof. The individual profit function

$$
\pi\left(y^{\prime}\right):=P_{\psi}\left((n-1) y+y^{\prime}, I\right) y^{\prime}-w l\left(y^{\prime}\right)
$$

is differentiable by (i). Using (ii) and (iii) it can be easily shown that $\pi^{\prime}\left(y^{\prime}\right)>0$ if $0 \leqslant y^{\prime}<y$ and that $\pi^{\prime}\left(y^{\prime}\right)<0$ if $y<y^{\prime} \leqslant \hat{y}(y)$. Therefore, $y$ is the unique maximum of $\pi$ on $[0, \hat{y}(y)]$.

Proof of Result 1. Define $\tilde{y}:=2 \cdot 3^{-(3+\sqrt{3}) /(4+2 \sqrt{3})} \bar{y}<\bar{y}$, choose any $y \in[\tilde{y}, \bar{y}]$ and define $p:=2 M /(n y)$. Since $\sigma \neq 1$ define $\beta:=4(1-p n(p-w)) /$ $(\sigma-1)$. $(y, p, n p y)$ is a stationary symmetric Cournot equilibrium relative to $w$ if the assumptions of Lemma 1 are satisfied for

$$
D_{\psi}\left(p^{\prime}, 2 M\right)=\frac{M}{p^{\prime}}\left(1+2 c\left(p^{\prime \beta} p^{-\beta}\right)\right),
$$

since the forecast function is $\psi\left(p^{\prime}\right)=p^{1+\beta} p^{-\beta}$. (iii) is fulfilled with equality by definition of $\beta$.

To check condition (i), use (1) to show that $D_{\psi}{ }^{\prime}(., 2 M)<0$ iff

$$
-1+2 c((1-c)(\sigma-1) \beta-1)<0 \quad \forall c \in(0,1) .
$$

But this can be shown to hold if $0<(\sigma-1) \beta<2+\sqrt{3}$ which follows from the assumptions on $n$ and $w$.

To show condition (ii), observe that $M R($.$) is strictly monotonically$ increasing if the elasticity of $D_{\psi}(., 2 M)$ is monotonically decreasing. Using (1) again and the observation that $c\left(p^{\prime \beta} p^{-\beta}\right)$ is monotonically increasing in $p^{\prime}$ since $(\sigma-1) \beta>0, D_{\psi}{ }^{\prime}\left(p^{\prime}, 2 M\right) p^{\prime} / D_{\psi}\left(p^{\prime}, 2 M\right)$ is monotonically decreasing in $p^{\prime}$ iff $2 c(1-c)(\sigma-1) \beta(1+2 c)^{-1}$ is monotonically decreasing in $c=c\left(p^{\prime \beta} p^{-\beta}\right)$. But this can be shown to hold only for $c>(\sqrt{3}-1) / 2$, which implies that the elasticity of $D_{\psi}(., 2 M)$ decreases monotonically for

$$
p^{\prime}>p c^{-1}((\sqrt{3}-1) / 2)^{1 / \beta}=p(\sqrt{3})^{1 /(\beta(1-\sigma))}=: \tilde{p} .
$$

A straightforward computation shows that

$$
\begin{aligned}
D_{\psi}(\tilde{p}, 2 M) & =n y(\sqrt{3})^{1+1 /(\beta \sigma-\beta)} / 2 \\
& \geqslant n y 3^{(3+\sqrt{3}) /(4+2 \sqrt{3})} / 2 \geqslant n \bar{y},
\end{aligned}
$$

which implies $\tilde{p} \leqslant p_{l}$. Therefore, $M R($.$) is strictly monotonically increasing$ on $\left[p_{l}, \infty\right)$.

As an illustration of Result 1, Fig. 1 displays the stationary equilibrium $(1,1,2)$ with unemployment for $n=2, w=0, \sigma=3, \beta=1$ and $M=1$. The labor capacity constraint $\bar{L}>2$ may not be too large, since marginal revenue becomes positive at large employment levels. 


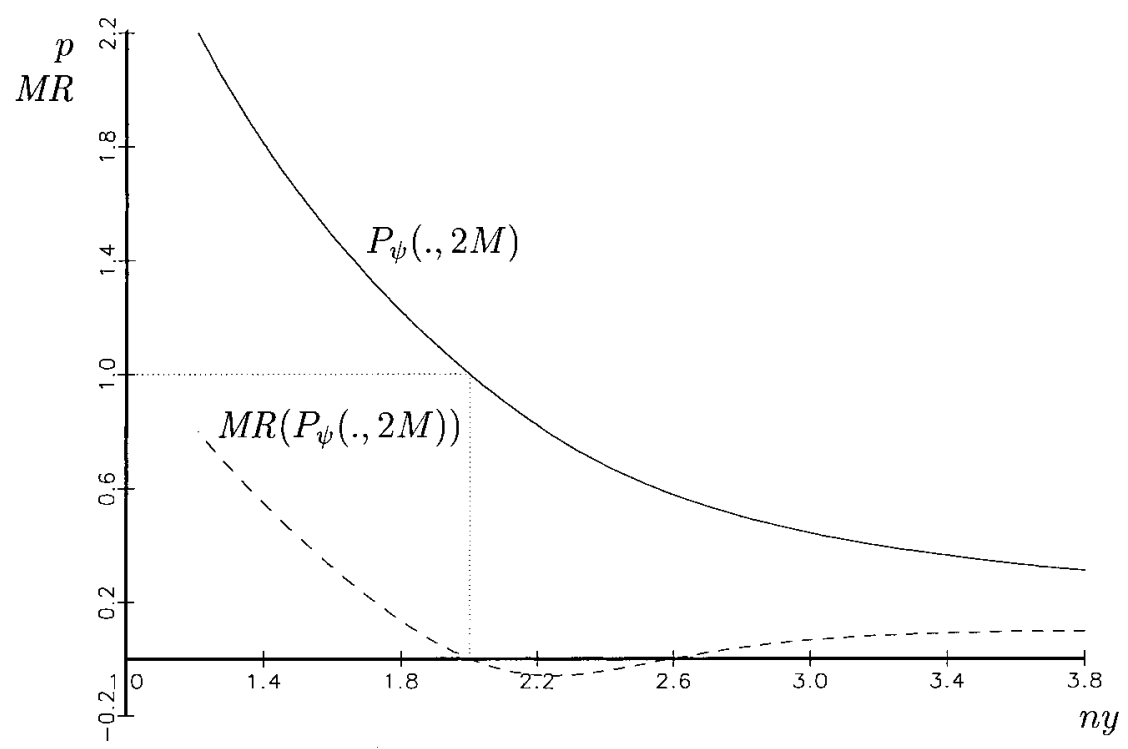

FIG. 1. Stationary equilibrium with unemployment at $w=0$.

It is important to notice that the existence of stationary equilibria with full employment and unemployment depends crucially on the expectations elasticity $\beta$. Consider again the first order condition (3) at a stationary equilibrium $(2 M /(n p), p, 2 M)$ :

$$
1-\frac{1}{4}(\sigma-1) \beta \geqslant \frac{p}{n(p-w)} \quad \text { and } \quad \frac{2 M}{p} \leqslant \bar{L} \quad \text { (one with equality). }
$$

Figure 2 illustrates the solutions $p$ of this condition as functions of $\beta$ given $\sigma>1$ and with $\beta^{*}:=4(1-1 / n) /(\sigma-1)$. The sets of stationary equilibria differ qualitatively for $w=0$ and for $w>0$ : while for $w=0$ and $\beta=\beta^{*}$ there is a continuum of candidates of stationary unemployment equilibria, for $\beta<\beta^{*}$ only full employment equilibria emerge. For $w>0$ there are unique stationary equilibria at different values of $\beta<\beta^{*}$ with unemployment if $\beta$ is close to $\beta^{*}$. If $w$ is larger than $2 M / \bar{L}$, there are only unemployment equilibria for all values of $\beta<\beta^{*}$. If $\beta>\beta^{*}$ there exists no stationary equilibrium.

In a model with multiple perfect foresight equilibria, it is reasonable to carry out an equilibrium selection with regard to some learning process. In the model considered here, it is impossible to study such a learning process globally, since it is hard to verify existence of temporary equilibria at a given forecast rule for each parameter specification, and an explicit iteration formula can not be computed. Instead one should try to analyze the 


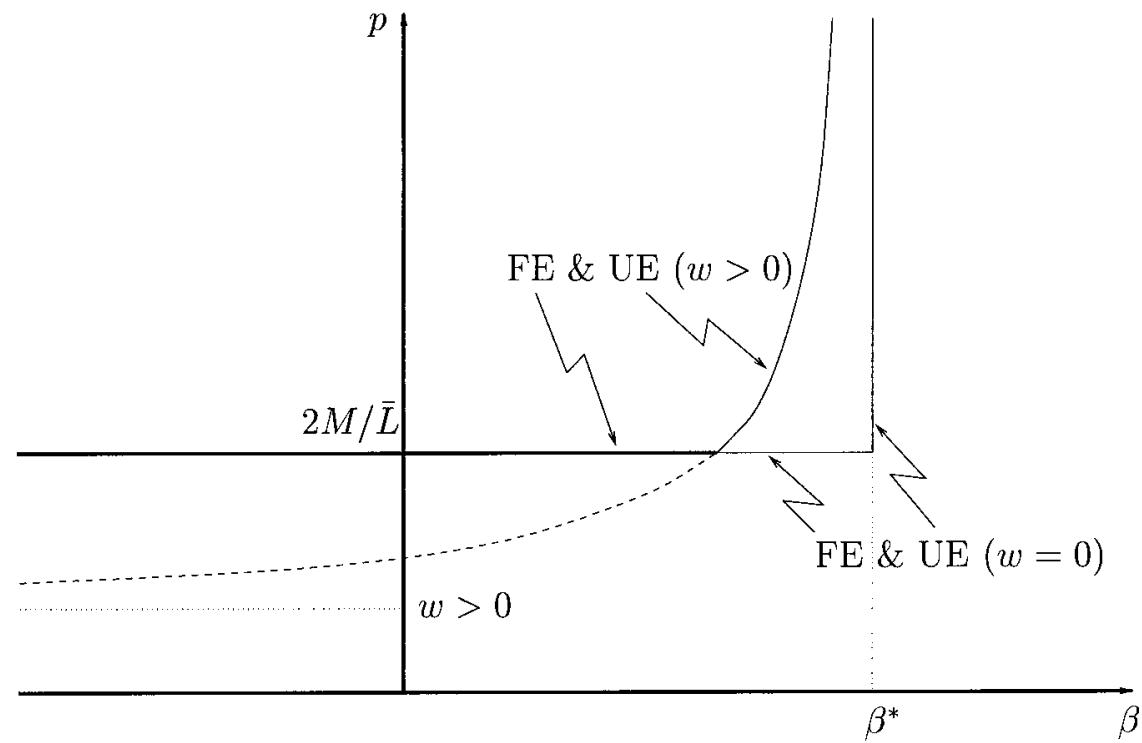

FIG. 2. Stationary full employment and unemployment equilibrium prices $p$ in dependence on the expectations elasticity $\beta$ for $w=0$ and $w>0$.

so-called local temporary equilibrium dynamics (see Grandmont [7]). We will describe such a process only for the constant elasticity rules defined above, even though an endogenous determination of $\beta$ (via OLS learning, for example) would be more relevant, since $\beta$ plays the decisive rule for the occurrence of unemployment in a stationary equilibrium.

The temporary equilibrium dynamics is described differently for unemployment and for full employment equilibria. In case of a stationary unemployment equilibrium $(y, p, I)$ consider

$$
\begin{aligned}
c\left(p_{t}^{\beta} \Phi_{t}\right)\left(1-c\left(p_{t}^{\beta} \Phi_{t}\right)\right)(\sigma-1) \beta & =1-\frac{p_{t}}{n\left(p_{t}-w\right)}, \quad \text { with } \\
n y_{t} & =\frac{M}{p_{t}\left(1-c\left(p_{t}^{\beta} \Phi_{t}\right)\right)}<\bar{L},
\end{aligned}
$$

where $\Phi_{t}=\left(p_{t}^{\mathrm{e}}\right)^{-\beta}$ in case of $(6), \Phi_{t}=\left(p_{t}^{\mathrm{e}}\right)^{1-\beta} p_{t-1}^{-1}$ in case of (7), and $\Phi_{t}=\Phi\left(p_{t-1}, \ldots, p_{t-\tau}\right)$ in case of $(8)$. The full employment temporary equilibrium dynamics is described by ${ }^{1}$

\footnotetext{
${ }^{1}$ The boundary case of two binding constraints in a stationary equilibrium will not be considered.
} 


$$
\begin{gathered}
\frac{M}{p_{t}\left(1-c\left(p_{t}^{\beta} \Phi_{t}\right)\right)}=\bar{L}, \quad \text { with } \\
c\left(p_{t}^{\beta} \Phi_{t}\right)\left(1-c\left(p_{t}^{\beta} \Phi_{t}\right)\right)(\sigma-1) \beta<1-\frac{p_{t}}{n\left(p_{t}-w\right)} .
\end{gathered}
$$

$p_{t}^{\mathrm{e}}, p_{t-1}, \ldots, p_{t-\tau}$ are assumed to be close to $p$, so that $\Phi_{t}$ is close to $p^{-\beta}$. If the assumptions of the above lemma are fulfilled for $\psi\left(p^{\prime}\right)=p^{\prime \beta+1} p^{-\beta}$, they are fulfilled also for $\psi\left(p^{\prime}\right)=p^{\prime \beta+1} \Phi_{t}$ with $\Phi_{t}$ close to $p^{-\beta}$ which implies that possible implicit solutions of (9) or (10) close enough to $p$ yield in fact temporary symmetric Cournot equilibria.

RESULT 2. Stationary unemployment equilibria relative to $w>0$ are stable under (6), (7), and (8). Stationary unemployment equilibria relative to $w=0$ are stable under (6) and (7), and are stable under (8) iff the corresponding price is stable under $p_{t}=\Phi\left(p_{t-1}, \ldots, p_{t-\tau}\right)^{-1 / \beta}$. Stationary full employment equilibria are stable under (6), and may be unstable under (7) and (8).

Proof. Consider first the case of a stationary unemployment equilibrium price $p$ relative to $w>0$. By the implicit function theorem (9) has a solution $p_{t}=\Gamma\left(\Phi_{t}\right)$ with $\Gamma^{\prime}\left(p^{-\beta}\right)=0$. This implies that $p$ is locally stable under (6), (7), and (8).

If $p$ is a stationary unemployment equilibrium price at $w=0, \beta=$ $4(1-1 / n) /(\sigma-1)$ is independent of $p$ and there is a continuum of stationary equilibria at $\beta$. (9) is then

$$
c\left(p_{t}^{\beta} \Phi_{t}\right)\left(1-c\left(p_{t}^{\beta} \Phi_{t}\right)\right)=4,
$$

which has the solution $p_{t}=\Phi_{t}^{-1 / \beta}$. Under (6) it follows $p_{t+1}^{\mathrm{e}}=p_{t}=p_{t}^{\mathrm{e}}$ so that the initially expected price level determines the stationary equilibrium. Under (7) it follows $p_{t+1}^{\mathrm{e}}=p_{t}=\left(p_{t}^{\mathrm{e}}\right)^{1-1 / \beta} p_{t-1}^{1 / \beta}$ so that the initial price and the initial expected price determine the stationary equilibrium.

If $p$ is a stationary full employment equilibrium price, the implicit function theorem gives a solution $p_{t}=\Gamma\left(\Phi_{t}\right)$ of $(10)$ iff $\beta(\sigma-1) \neq 2$, and it is $\Gamma^{\prime}\left(p^{-\beta}\right)=p^{1+\beta}(\sigma-1) /(2-\beta(\sigma-1))$. For $(6)$, it is

$$
\left.\frac{d p_{t+1}^{\mathrm{e}}}{d p_{t}^{\mathrm{e}}}\right|_{p_{t}^{\mathrm{e}}=p}=-\frac{\beta(\sigma+1)}{2-\beta(\sigma-1)} \in[0,1)
$$

since $\beta \in(-1,0]$. In case of (7) and (8) the local dynamics can be unstable since $\Gamma^{\prime}\left(p^{-\beta}\right)$ can be arbitrary large for $\beta(\sigma-1)$ close to 2. For example, in case of (7) the local dynamics is described by $q_{t+1}=\Gamma\left(q_{t}\right)^{-\beta^{2}} q_{t}^{1-\beta}$ with 
$q_{t}=\left(p_{t}^{\mathrm{e}}\right)^{1-\beta} p_{t-1}^{-1}$. The derivative at the steady state $q=p^{-\beta}$ is $1-2 \beta /(2-\beta(\sigma-1))$ and may be larger than 1 or less than -1 .

Notice that such a strong stability result for unemployment equilibria needs not to hold if some assumptions are relaxed. Even the introduction of a discount factor different from one or a non-linear technology would imply $\Gamma^{\prime}\left(p^{-\beta}\right) \neq 0$ generally, so that stability can no longer be guaranteed. In this respect, Result 2 should only be understood as an example showing that for one plausible model specification simple learning mechanisms lead to stable unemployment equilibria, while full employment equilibria are unstable.

\section{SUMMARY AND CONCLUSION}

It has been shown that generically a continuum of equilibria with perfect foresight and unemployment at arbitrary low wages exists in overlapping generations models with Cournot competition on the goods market. This result differs from the result of Schultz who excludes involuntary unemployment under the hypothesis of inelastic price expectations. It differs as well from competitive overlapping generations models where in general only finitely many stationary equilibria exist. ${ }^{2}$ However, as the number of firms tends to infinity, all equilibria shrink to a small interval converging to the full employment allocation.

The crucial reason for this multiplicity phenomenon is the role of different forecast functions that are all consistent with perfect foresight, but lead to different demand functions against which oligopolists maximize. This may not seem too surprising, since it is closely related to results of Böhm [2] and Grodal [8], who show that in general equilibrium models different price normalization rules lead to almost arbitrary Cournot equilibria. In an intertemporal context with price of money normalized to one, price expectations play the role of a normalization rule, and should therefore influence equilibrium allocations.

At hand of an example it was argued that stationary unemployment equilibria may be stable under the local temporary equilibrium dynamics, while full employment equilibria are not. However, this result is not suited as an equilibrium selection, since it is very special and since it does not explain how the crucial forecast elasticity parameter is determined. Furthermore, a global learning process can not be described in this setting. It seems more promising to study global learning processes by allowing firms not to know the objective demand function, but to estimate it instead

\footnotetext{
${ }^{2}$ An exception is the case of stochastic OLG models with incomplete asset structure (Gottardi [6]).
} 
from past data, even though such a subjective demand approach usually leads to a larger set of correlated equilibria.

\section{REFERENCES}

1. R. Abraham, J. Marsden, and T. Ratiu, "Manifolds, Tensor Analysis, and Applications," Addison-Wesley, London, 1983.

2. V. Böhm, The foundation of the theory of monopolistic competition revisited, J. Econ. Theory 63 (1994), 208-218.

3. G. Bonanno, General equilibrium theory with imperfect competition, J. Econ. Surveys 4 (1990), 297-328.

4. C. D'Aspremont, R. Dos Santos Ferreira, and L. A. Gérard-Varet, Imperfect competition, rational expectations, and unemployment, in "Equilibrium Theory and Applications: Proceedings of the Sixth International Symposium in Economic Theory and Econometrics" (Barnett et al., Eds.), Cambridge Univ. Press, Cambridge, 1991.

5. P. Dehez, Monopolistic equilibrium and involuntary unemployment, J. Econ. Theory 36 (1985), 160-165.

6. P. Gottardi, Stationary monetary equilibria in overlapping generations models with incomplete markets, J. Econ. Theory 71 (1996), 75-89.

7. J. M. Grandmont, Expectations formation and stability of large socioeconomic systems, Econometrica, to appear.

8. B. Grodal, Profit maximization and imperfect competition, in "Economics in a Changing World" (B. Allen, Ed.), Vol. 2, MacMillan \& Co., London, 1996.

9. O. Hart, A model of imperfect competition with Keynesian features, Quart. J. Econ. 97 (1982), 109-138.

10. M. Lettau and T. Van Zandt, "Robustness of Adaptive Expectations as an Equilibrium Selection Device," Center for Economic Research Discussion Paper 9598, Tilburg University, 1995.

11. A. Marcet and T. Sargent, Convergence of least squares learning mechanisms in selfreferential linear stochastic models, J. Econ. Theory 48 (1989), 337-368.

12. N. Rankin, Imperfect competition, expectations and the multiple effects of monetary growth, Econ. J. 102 (1992), 743-753.

13. C. Schultz, The impossibility of involuntary unemployment in an overlapping generations model with rational expectations, J. Econ. Theory 58 (1992), 61-76.

14. J. Silvestre, The market-power foundations of macroeconomic policy, J. Econ. Lit. 31 (1993), 105-141.

15. J. Silvestre, Market power in macroeconomic models: new developments, Ann. d'Econ. Statist. 37/38 (1995), 319-356. 